\title{
Effects of the pro-inflammatory milieu on the dedifferentiation of cultured fibroblast-like synoviocytes
}

\author{
HONG SEO CHOI ${ }^{1 *}$, CHUN JEIH RYU $^{1 *}$, HYUN MI CHOI ${ }^{2}$, JIN SUNG PARK ${ }^{3}$, JAE-HOON LEE ${ }^{4}$, \\ KANG IL KIM ${ }^{4}$, HYUNG-IN YANG ${ }^{2}$, MYUNG CHUL YOO ${ }^{4}$ and KYOUNG SOO KIM ${ }^{2}$ \\ ${ }^{1}$ Institute of Bioscience, Department of Bioscience and Biotechnology, Sejong University, Seoul 143-747; \\ ${ }^{2}$ East-West Bone and Joint Research Institute, Kyung Hee University Hospital at Gangdong, \\ Kyung Hee University Medical School, Seoul 134-727; ${ }^{3}$ Department of Anatomy, Ajou University, School of Medicine, \\ Suwon 443-721; ${ }^{4}$ Department of Orthopedic Surgery, Kyung Hee University Hospital at Gangdong, \\ Kyung Hee University Medical School, Seoul 134-727, Republic of Korea
}

Received October 18, 2011; Accepted January 13, 2012

DOI: $10.3892 / \mathrm{mmr} .2012 .767$

\begin{abstract}
The aim of this study was to determine whether the inflammatory milieu and/or hypoxia induces the dedifferentiation of synovial cells into mesenchymal stem-like cells, which may contribute to the tumor-like growth of synovial cells. Expression of mesenchymal stem cell markers (CD24, CD44, CD90, CD106, CD146 and Stro-1) was compared among cultured fibroblast-like synoviocytes (FLSs) from patients with rheumatoid arthritis (RA) or osteoarthritis (OA), bone marrow mesenchymal stem cells (BM MSCs) and normal dermal fibroblasts. After the cells were stimulated with pro-inflammatory cytokines for 3 days under hypoxia or normoxia, the stem cell markers were analyzed by FACS. CD44 and CD90 were expressed constitutively in all four cell types. Only the BM MSCs strongly expressed CD146. The expression of stem cell markers was similar between FLSs from RA and those from OA patients. In addition, the expression levels in FLSs were similar to those in normal dermal fibroblasts. The stimulation of FLSs and dermal fibroblasts with IL-1 $\beta$ or a mixture of cytokines under hypoxia did not induce a marked change in the expression of stem cell markers. These results indirectly suggest that the pro-inflammatory milieu may be not sufficient to induce the dedifferentiation of FLSs in arthritic joints.
\end{abstract}

Correspondence to: Dr Kyoung Soo Kim, East-West Bone and Joint Research Institute, Kyung Hee University Hospital at Kangdong, Seoul 134-727, Republic of Korea

E-mail: labrea46@yahoo.co.kr

"Contributed equally

Key words: dedifferentiation, fibroblast-like synoviocytes, rheumatoid arthritis, osteoarthritis, bone marrow mesenchymal stem cell, dermis, stem cell markers

\section{Introduction}

Rheumatoid arthritis (RA) is characterized by the proliferation of fibroblast-like synoviocytes (FLSs) in affected joints. The onset of RA causes dramatic morphological changes in the synovial membrane, including thickening of the intimal lining. In addition, the FLSs in RA produce various cytokines and chemokines that recruit more immune cells into the joint cavities, and immune cells infiltrate into the proliferating synovial membranes, forming a pannus that exhibits aggressive, tumor-like growth and invades and erodes the cartilage and subchondral bone (1). The FLSs in the pannus resemble immature, transformed fibroblasts and are, in many ways, different from normal FLSs (e.g., invasiveness and cartilage degradation) $(2,3)$. The mechanisms underlying the tumor-like phenotype of FLSs in RA are poorly understood. Many attempts have been made to explain the phenotype by dedifferentiation of normal FLSs, presumably through an accumulation of genetic and epigenetic abnormalities similar to cancer (4). A number of proto-oncogenes, including myc, ras and fos, are overexpressed in the FLSs of RA (5), but no common genetic abnormalities have been identified in these genes. Somatic mutations in the tumor-suppressor gene p53 are associated with RA (6), but a role for these mutations in the 'pseudotransformed' FLS phenotype is not known. Slowing of the rapid growth of FLSs after passage in culture is also against the 'transformation' hypothesis (7).

The pannus is comprised of many cell types, including inflammatory, immune and mesenchymal cells. A fraction of the heterogeneous FLS population in RA was recently found to have properties usually associated with mesenchymal stem cells (MSCs). When appropriately stimulated in culture, a proportion of the FLSs differentiates into chondrocytes, osteoblasts, adipocytes and muscle cells $(8,9)$. Animal studies have shown that bone marrow-derived mesenchymal cells (BM MSCs) appear to contribute to synovial proliferation $(10,11)$. By contrast, local inflammatory cytokines suppress the differentiation of MSCs in RA-affected joints $(12,13)$. Thus, arthritic FLSs have been suggested 
to contain a substantial fraction of BM MSCs, but they are under-differentiated because of the inflammatory milieu in arthritic joints (14). Inflammatory milieu and hypoxia are characteristic of arthritic joints. The inflammatory milieu may induce the dedifferentiation of terminally differentiated cells (e.g., chondrocytes) in arthritic joints (15). In addition, inflammation-induced hypoxia may induce the redifferentiation of dedifferentiated chondrocytes (16).

In this study, to better understand the tumor-like phenotypes of arthritic FLSs, we investigated whether inflammatory stimuli and hypoxia in arthritic joints induce the dedifferentiation of FLSs into mesenchymal stem-like cells, and whether the dedifferentiated FLSs contribute to the tumor-like phenotypes of RA FLSs. We compared the expression of MSC surface markers on fibroblasts from RA and osteoarthritis (OA) patients, dermal fibroblasts and bone marrow. In addition, we examined whether synovial or dermal fibroblasts exposed to inflammatory stimuli and hypoxia have altered expression of MSC surface markers.

\section{Materials and methods}

Cell culture. All in vitro experiments were carried out with FLSs derived from patients with RA or OA who met the 1987 American College of Rheumatology criteria for diagnosis, had been treated with non-biological disease-modifying antirheumatic drugs (DMARDs), and underwent therapeutic joint surgery. The synovial tissues were collected from patients after obtaining informed consent. The FLSs were isolated and grown in Dulbecco's modified essential medium (DMEM; low glucose; Invitrogen, Carlsbad, CA, USA), supplemented with $10 \%(\mathrm{v} / \mathrm{v})$ fetal bovine serum (FBS; Invitrogen) and $1 \mathrm{X}$ antibiotic-antimycotic (Invitrogen).

Normal dermal fibroblasts were purchased from Modern Cell and Tissue Technologies (Seoul, Korea) and cultured in DMEM (high glucose). When the cells had grown to confluence, they were detached using cell dissociation buffer (Invitrogen) and split at a 1:3 ratio. FLSs from 3 patients at passage 3 were used in all experiments.

To test the effect of pro-inflammatory cytokines on dedifferentiation of cells, the cells were cultured for 3 days in the presence or absence of pro-inflammatory cytokines. For hypoxic effect, hypoxic conditions were generated by incubating the cells at $2 \% \mathrm{O}_{2}$ in a hypoxic chamber gassed with a combination of $\mathrm{N}_{2}$ and $\mathrm{CO}_{2}$ (Invivo2 200; Ruskinn Technology Ltd., Pencoed, UK).

BM MSCs were purchased from Innomedi (Seoul, Korea). The BM MSCs were expanded for 9-11 days in 100-mm culture dishes, and then re-plated in 150-mm culture dishes with $0.25 \%$ trypsin-EDTA (Invitrogen) at a density of $5 \times 10^{5}$ cells. FACS analysis was performed after culturing for 15 days. This study was approved by the Institutional Review Board for Human Research of Kyung Hee University Hospital at Kangdong.

FACS analysis of stem cell markers. The FLSs were treated with cell dissociation buffer (Invitrogen) for $20 \mathrm{~min}$ in a $37^{\circ} \mathrm{C}$ incubator and filtered through a $40-\mu \mathrm{m}$ cell strainer. The dissociated cells $\left(2 \times 10^{5}\right.$ cells) were resuspended immediately in PBA (1\% bovine serum albumin, $0.02 \% \mathrm{NaN}_{3}$ in PBS, pH 7.4) and incubated with phycoerythrin-conjugated anti-CD24
(BD Biosciences, Seoul, Korea), anti-CD44 (BD Biosciences), anti-CD90 (BD Biosciences), anti-CD106 (BD Biosciences), anti-CD146 (BD Biosciences) and anti-STRO-1 (Santa Cruz Biotechnology, Santa Cruz, CA, USA) for $30 \mathrm{~min}$ at $4^{\circ} \mathrm{C}$. After washing with PBA, the propidium iodide (PI)-negative cells were analyzed for antibody binding using FACSCalibur (BD Biosciences) and Cell Quest software (BD Biosciences).

\section{Results}

The MSC surface markers CD44 and CD90 were expressed constitutively in all four cell types (Fig. 1). Only BM MSCs expressed CD146. None of the cells expressed CD24. The expression in FLSs from RA patients was distinctly different from that of normal dermal fibroblasts. Unexpectedly, the expression of stem cell markers in FLSs from RA patients was similar to that of FLSs from OA patients. One reason for the lack of difference may be the in vitro cell culture. The expression of stem cell markers may disappear during cell culture under conditions lacking inflammation. To evaluate this limitation, the FLSs from OA patients and normal dermal fibroblasts were cultured for $72 \mathrm{~h}$ in the presence or absence of $10 \mathrm{ng} / \mathrm{ml} \mathrm{IL-1} \beta$ and the stem cell markers were compared to those of unstimulated FLSs. As shown in Fig. 2, the expression of CD24, CD44 and CD90 was similar regardless of IL-1 $\beta$ stimulation. The expression of CD106, CD146 and Stro-1 was also not significantly different in response to IL- $1 \beta$ stimulation. Next, we investigated whether the expression pattern was affected when the cells were cultured under hypoxic conditions $\left(2 \% \mathrm{O}_{2}\right.$ concentration), which is one of the factors affecting the expression of various genes in inflammatory arthritic joints. The FLSs from RA patients were cultured for $72 \mathrm{~h}$ with IL-1 $\beta(10 \mathrm{ng} / \mathrm{ml})$ or a combination of cytokines (1 ng/ml each IL-1 $\beta$, TNF- $\alpha$, IL-6, IL-17, IL-22 and IL-23) in the presence or absence of hypoxia (Fig. 3). The MSC markers in the RA FLSs were not significantly different in the presence of pro-inflammatory stimulation or under hypoxic conditions.

\section{Discussion}

We investigated whether the MSC markers in FLSs from RA patients indicated dedifferentiation into MSCs in response to pro-inflammatory cytokines or hypoxic conditions, as this dedifferentiation may contribute to the tumor-like growth of synoviocytes in RA during progressive inflammation. Thus, we compared the expression of MSC surface markers (CD24, CD44, CD90, CD106, CD146 and Stro-1) on FLSs from RA or OA patients, dermal fibroblasts and BM MSCs. However, we did not identify any significant changes in response to pro-inflammatory stimulation or hypoxic conditions.

Extensive analyses have identified MSC surface markers in rat, mice and humans (17-20). Human MSCs have been reported to be positive for CD44, CD90 and CD105, and negative for CD14, CD34 and CD45 (20). Other cell markers, such as CD106, CD146 and Stro-1, are also expressed on the surface of MSCs (21), but their expression patterns change depending on the culture conditions or specific types of stimulation (22-24). Surface markers are also down-regulated with passaging (25). By contrast, the stem cell markers in chondrocyte dedifferentiation have been employed in autologous 

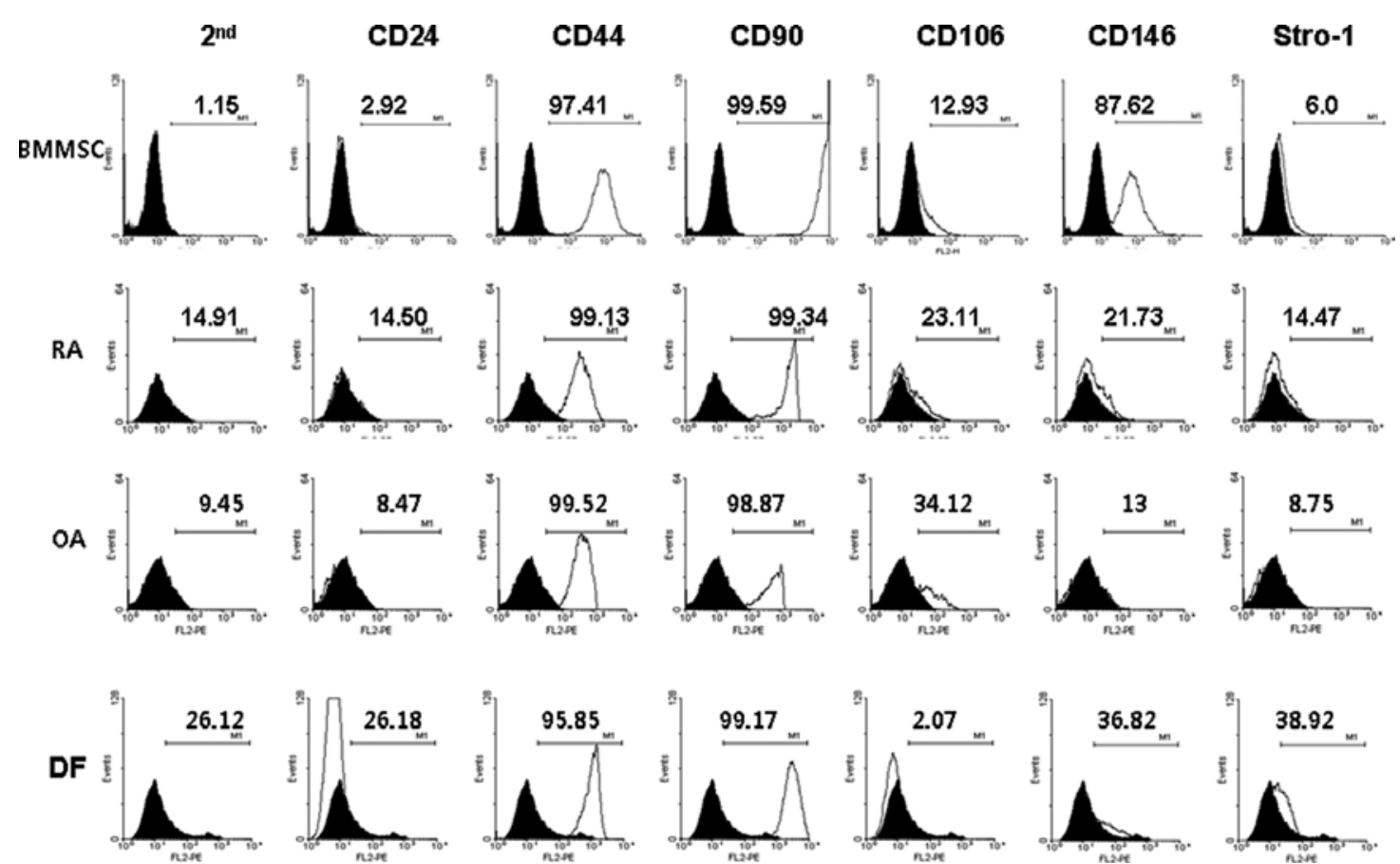

Figure 1. FACS comparison of the stem cell markers in fibroblast-like synoviocytes (FLSs) from rheumatoid arthritis (RA) or osteoarthritis (OA) patients, normal dermal fibroblasts (DF) and bone marrow mesenchymal stem cells (BMMSC). The data shown are representative of three independent experiments with similar results.
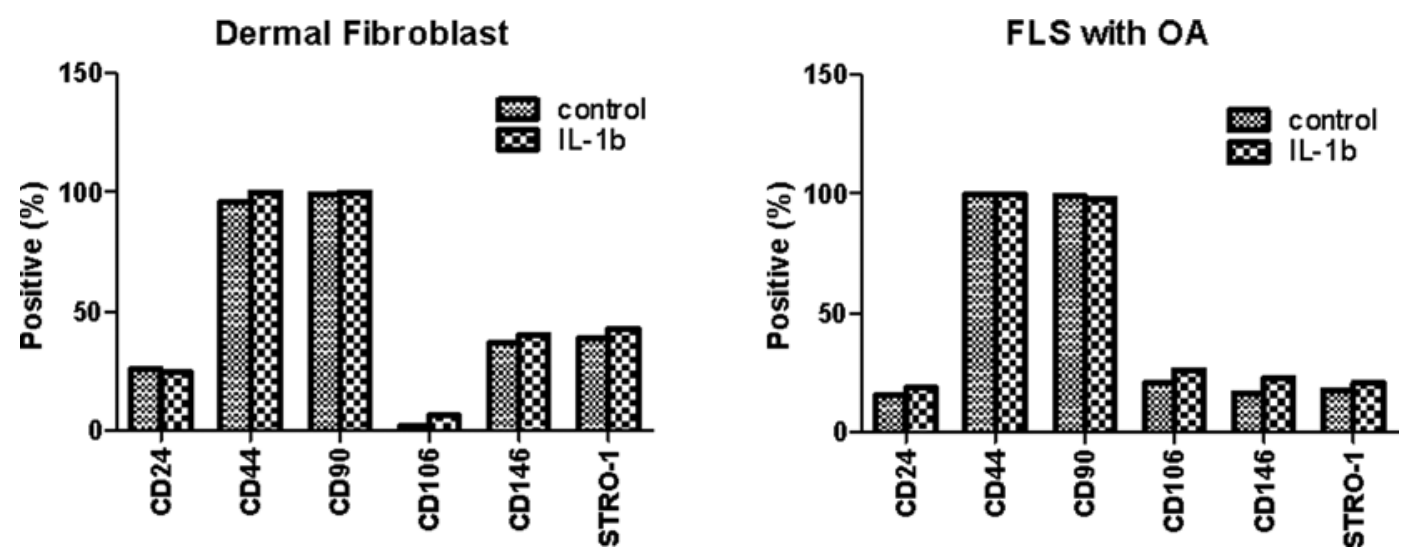

Figure 2. Effect of IL-1 $\beta$ on stem cell markers in fibroblast-like synoviocytes (FLSs) from osteoarthritis (OA) patients and normal dermal fibroblasts. The cells were cultured for 3 days in the presence or absence of IL-1 $(10 \mathrm{ng} / \mathrm{ml})$. The surface markers were analyzed by FACS. The data shown are representative of three independent experiments with similar results.
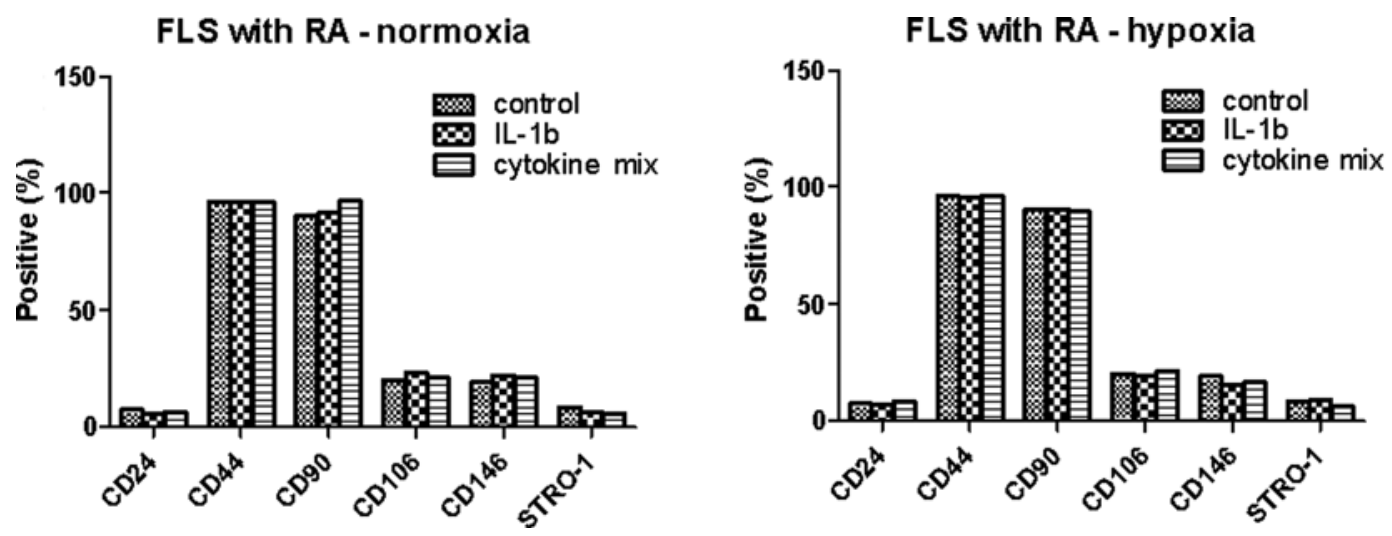

Figure 3. Combined effect of IL-1 $\beta$ and hypoxia on stem cell markers in fibroblast-like synoviocytes (FLSs) from rheumatoid arthritis (RA) patients. The cultured cells from RA patients were incubated with IL-1 $(10 \mathrm{ng} / \mathrm{ml})$ or the mixture of various pro-inflammatory cytokines (IL-1 $\beta$, TNF- $\alpha$, IL-6, IL-17, IL-22 and IL-23; $1 \mathrm{ng} / \mathrm{ml}$ of each) under normoxia or hypoxia. The data shown are representative of three independent experiments with similar results. 
chondrocyte implantation (26). Chondrocytes isolated from a small biopsy of hyaline cartilage are expanded ex vivo, and once a sufficient number of cells are obtained, the chondrocytes are then implanted in the cartilage defect. However, the proliferating chondrocytes gradually lose their differentiated phenotype. These phenotypic changes lead to the production of an extracellular matrix with inferior biomechanical properties. For tissue, such as cartilage, high quality biomechanical properties are critical. Therefore, investigating parameters that favor the redifferentiation of dedifferentiated chondrocytes before implantation is critical. Thus, the stem cell markers are investigated to determine phenotypic changes. However, the stem cell marker changes in FLSs in RA during FLS dedifferentiation have been rarely studied.

In this study, we attempted to find the parameters or conditions that favor the dedifferentiation of FLSs, which may lead to being able to control or inhibit the 'tumor-like growth' of the synovial membrane of patients with RA. However, the in vitro system in this study has limitations. Thus, we tried to mimic the in vivo pro-inflammatory conditions, yet it was still likely not enough to meet the in vivo conditions. In addition, our in vitro systems may impair the dedifferentiation of FLSs since the differentiation potential of cultured cells decreases after every passage $(27,28)$. Thus, the cell surface markers may not have been significantly altered by the pro-inflammatory cytokines and/or hypoxia due to unknown factors in the cell culture. We also examined whether cell passage affects the expression of stem cell markers. The stem cell markers of FLSs at the second passage were compared to those of the sixth passage when the FLSs were prepared at the same time. Cell passage did not appear to affect the expression pattern of the stem cell markers in FLSs, even in response to cytokine stimulation (data not shown). In conclusion, these results indirectly suggest that the pro-inflammatory milieu may not induce the dedifferentiation of FLSs in arthritic joints.

\section{Acknowledgements}

This study was supported by the National Research Foundation of Korea (2011-0009061 to KSK; 2011-0002659 and 2011-0027795 to CJR).

\section{References}

1. Firestein GS: Evolving concepts of rheumatoid arthritis. Nature 423: 356-361, 2003

2. Pap T, Muller-Ladner U, Gay RE and Gay S: Fibroblast biology Role of synovial fibroblasts in the pathogenesis of rheumatoid arthritis. Arthritis Res 2: 361-367, 2000.

3. Edwards JC: Fibroblast biology. Development and differentiation of synovial fibroblasts in arthritis. Arthritis Res 2: 344-347, 2000

4. Firestein GS: Invasive fibroblast-like synoviocytes in rheumatoid arthritis. Passive responders or transformed aggressors? Arthritis Rheum 39: 1781-1790, 1996.

5. Muller-Ladner U, Kriegsmann J, Gay RE and Gay S: Oncogenes in rheumatoid arthritis. Rheum Dis Clin North Am 21: 675-690, 1995.

6. Yamanishi Y, Boyle DL, Rosengren S, Green DR, Zvaifler NJ and Firestein GS: Regional analysis of p53 mutations in rheumatoid arthritis synovium. Proc Natl Acad Sci USA 99: 10025-10030, 2002.

7. Lafyatis R, Remmers EF, Roberts AB, Yocum DE, Sporn MB and Wilder RL: Anchorage-independent growth of synoviocytes from arthritic and normal joints. Stimulation by exogenous platelet-derived growth factor and inhibition by transforming growth factor-beta and retinoids. J Clin Invest 83: 1267-1276, 1989.
8. Yamasaki S, Nakashima T, Kawakami A, et al: Cytokines regulate fibroblast-like synovial cell differentiation to adipocytelike cells. Rheumatology (Oxford) 43: 448-452, 2004.

9. Zvaifler NJ, Tsai V, Alsalameh S, von Kempis J, Firestein GS and Lotz M: Pannocytes: distinctive cells found in rheumatoid arthritis articular cartilage erosions. Am J Pathol 150: 1125-1138, 1997.

10. Nakagawa S, Toritsuka Y, Wakitani S, et al: Bone marrow stromal cells contribute to synovial cell proliferation in rats with collagen induced arthritis. J Rheumatol 23: 2098-2103, 1996.

11. Marinova-Mutafchieva L, Williams RO, Funa K, Maini RN and Zvaifler NJ: Inflammation is preceded by tumor necrosis factordependent infiltration of mesenchymal cells in experimental arthritis. Arthritis Rheum 46: 507-513, 2002.

12. Murakami S, Lefebvre V and de Crombrugghe B: Potent inhibition of the master chondrogenic factor Sox 9 gene by interleukin-1 and tumor necrosis factor-alpha. J Biol Chem 275: 3687-3692, 2000.

13. Gilbert L, He X, Farmer P, et al: Expression of the osteoblast differentiation factor RUNX2 (Cbfa1/AML3/Pebp2alpha A) is inhibited by tumor necrosis factor-alpha. J Biol Chem 277: 2695-2701, 2002.

14. Li X and Makarov SS: An essential role of NF-kappaB in the 'tumor-like' phenotype of arthritic synoviocytes. Proc Natl Acad Sci USA 103: 17432-17437, 2006

15. Wehling N, Palmer GD, Pilapil C, et al: Interleukin-1beta and tumor necrosis factor alpha inhibit chondrogenesis by human mesenchymal stem cells through NF-kappaB-dependent pathways. Arthritis Rheum 60: 801-812, 2009.

16. Duval E, Leclercq S, Elissalde JM, Demoor M, Galera P and Boumediene K: Hypoxia-inducible factor 1alpha inhibits the fibroblast-like markers type I and type III collagen during hypoxia-induced chondrocyte redifferentiation: hypoxia not only induces type II collagen and aggrecan, but it also inhibits type I and type III collagen in the hypoxia-inducible factor lalpha-dependent redifferentiation of chondrocytes. Arthritis Rheum 60: 3038-3048, 2009

17. Javazon EH, Colter DC, Schwarz EJ and Prockop DJ: Rat marrow stromal cells are more sensitive to plating density and expand more rapidly from single-cell-derived colonies than human marrow stromal cells. Stem Cells 19: 219-225, 2001.

18. Eslaminejad MB, Nikmahzar A, Taghiyar L, Nadri S and Massumi M: Murine mesenchymal stem cells isolated by low density primary culture system. Dev Growth Differ 48: 361-370, 2006.

19. Docheva D, Popov C, Mutschler W and Schieker M: Human mesenchymal stem cells in contact with their environment: surface characteristics and the integrin system. J Cell Mol Med 11: 21-38, 2007

20. Pittenger MF, Mackay AM, Beck SC, et al: Multilineage potential of adult human mesenchymal stem cells. Science 284: 143-147, 1999.

21. Steinert AF, Kunz M, Prager P, et al: Mesenchymal stem cell characteristics of human anterior cruciate ligament outgrowth cells. Tissue Eng Part A 17: 1375-1388, 2011.

22. Yang JW, de Isla N, Huselstein C, et al: Evaluation of human MSCs cell cycle, viability and differentiation in micromass culture. Biorheology 43: 489-496, 2006.

23. Wiesmann A, Buhring HJ, Mentrup C and Wiesmann HP: Decreased CD90 expression in human mesenchymal stem cells by applying mechanical stimulation. Head Face Med 2: 8, 2006.

24. Honczarenko M, Le Y, Swierkowski M, Ghiran I, Glodek AM and Silberstein LE: Human bone marrow stromal cells express a distinct set of biologically functional chemokine receptors. Stem Cells 24: 1030-1041, 2006.

25. Halfon S, Abramov N, Grinblat B and Ginis I: Markers distinguishing mesenchymal stem cells from fibroblasts are downregulated with passaging. Stem Cells Dev 20: 53-66, 2011.

26. Lee HJ, Choi BH, Min BH and Park SR: Changes in surface markers of human mesenchymal stem cells during the chondrogenic differentiation and dedifferentiation processes in vitro. Arthritis Rheum 60: 2325-2332, 2009.

27. Digirolamo CM, Stokes D, Colter D, Phinney DG, Class R and Prockop DJ: Propagation and senescence of human marrow stromal cells in culture: a simple colony-forming assay identifies samples with the greatest potential to propagate and differentiate. Br J Haematol 107: 275-281, 1999.

28. Sekiya I, Colter DC and Prockop DJ: BMP-6 enhances chondrogenesis in a subpopulation of human marrow stromal cells. Biochem Biophys Res Commun 284: 411-418, 2001. 